\title{
One-Pot Glyco-Affinity Precipitation Purification for Enhanced Proteomic: The Flexible Alignment of Solution-Phase Capture/Release and Solid-Phase Separation
}

\author{
Xue-Long Sun,* Carolyn Haller, XiaoYi Wu, Vincent P. Conticello, and Elliot L. Chaikof*
}

Departments of Surgery and Biomedical Engineering, Emory University School of Medicine and Georgia Institute of Technology, Atlanta, Georgia 30322 and School of Chemical

Engineering, Georgia Institute of Technology, Atlanta, Georgia 30332

E-mail: xsun@emory.edu or echaiko@emory.edu

\section{Supporting Information}

\section{EXPERIMENTAL SECTIONS}

Materials. All solvents and reagents were purchased from commercial sources and were used as received, unless otherwise noted. 2-Azidoethanol was synthesized as described as literature. ${ }^{1)}$

Methods. Thin-layer chromatography (TLC) was performed on Whatman silica gel aluminum backed plates of $250 \mu \mathrm{m}$ thickness on which spots were visualized with UV light or charring the plate after dipping in $10 \% \mathrm{H}_{2} \mathrm{SO}_{4}$ in Methanol. Mass spectra (MS/FAB) were obtained at an ionizing voltage of $70 \mathrm{eV} .{ }^{1} \mathrm{H}$ spectra were recorded at room temperature with a Varian INOVA 400 and 600 spectrometer.

Synthesis of 2-aminoethyl $\beta$-glucoside and 2-aminoethyl $\beta$-lactoside

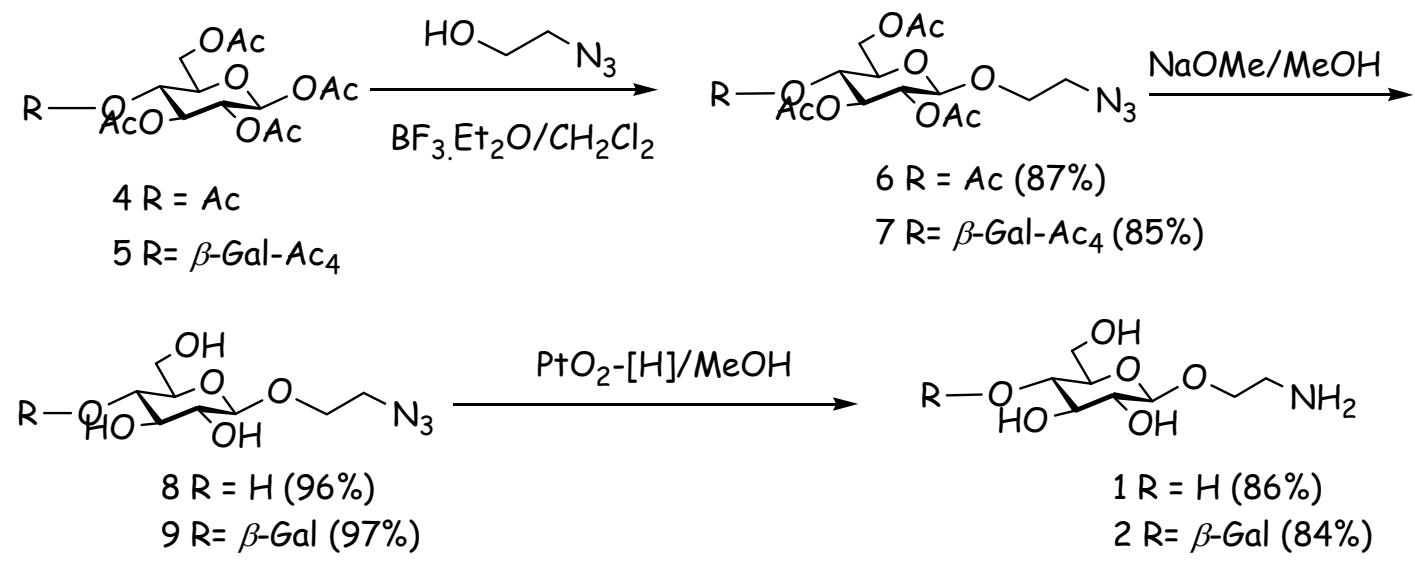

2-Azidoethyl 4- $O$-(2,3,4,6-tetra- $O$-acetyl- $\beta$-D-galatopyranosyl)-2,3,6-tri- $O$-acetyl- $\beta$-Dglucopyranoside (7). To a cooled (ice-water bath), stirred solution of peracetyl-lactose 5 (5.0 g, 7.37 
mmol), 2-azidoethanol ${ }^{1)}(0.770 \mathrm{~g}, 8.85 \mathrm{mmol}, 1.2 \mathrm{eq})$ in dichloromethane $(20 \mathrm{~mL})$ was added $\mathrm{BF}_{3}-$ etherate $(5.198 \mathrm{~g}, 36.87 \mathrm{mmol}, 5 \mathrm{eq})$. The reaction mixture was stirred for $1 \mathrm{hr}$ at $0{ }^{\circ} \mathrm{C}$ and then for 12 hrs at room temperature under an Ar atmosphere. The mixture was diluted with chloroform $(20 \mathrm{~mL})$, washed with cold water $(20 \mathrm{~mL})$ and aqueous sodium hydrogen carbonate $(20 \mathrm{~mL})$, and filterd. The filtrate was evaporated to give a residue, which was purified by column chromatography $\left(\mathrm{SiO}_{2}\right)$ using acetone- $n$-hexane $(2: 3)$ as eluent to afford 7 (4.420 g, 85\%). ${ }^{1} \mathrm{H} \mathrm{NMR}\left(\mathrm{CDCl}_{3}\right) \delta$ : 5.34 (br.d, $1 \mathrm{H}, J=$ $3.0 \mathrm{~Hz}), 5.19(\mathrm{t}, 1 \mathrm{H}, J=9.3 \mathrm{~Hz}), 5.10(\mathrm{dd}, 1 \mathrm{H}, J=10.5,7.8 \mathrm{~Hz}), 4.94(\mathrm{dd}, 1 \mathrm{H}, J=10.5,3.3 \mathrm{~Hz}), 4.91$ $(\mathrm{dd}, 1 \mathrm{H}, J=9.6,7.8 \mathrm{~Hz}), 4.55(\mathrm{~d}, 1 \mathrm{H}, J=8.1 \mathrm{~Hz}), 4.48(\mathrm{~d}, 1 \mathrm{H}, J=7.5 \mathrm{~Hz}), 4.15-4.03$ (m, $2 \mathrm{H}), 4.00$ $3.95(\mathrm{~m}, 1 \mathrm{H}), 3.86(\mathrm{t}, 1 \mathrm{H}, J=6.6 \mathrm{~Hz}), 3.81(\mathrm{t}, 1 \mathrm{H}, J=9.3 \mathrm{~Hz}), 3.70-3.58(\mathrm{~m}, 2 \mathrm{H}), 3.50-3.42$ (m. 1 $\mathrm{H})$, 3.29-3.22 (m, $1 \mathrm{H})$. HR-MS (FAB) calcd. For $\mathrm{C}_{28} \mathrm{H}_{39} \mathrm{O}_{18} \mathrm{~N}_{3} \mathrm{Li} 712.2406$ found 712.2398 [M+Li] ${ }^{+}$.

2-Azidoethyl -2,3,4,6-tetra- $\boldsymbol{O}$-acetyl- $\boldsymbol{\beta}$-D-glucopyranoside (6) was synthesized from peracetyl glucose 4 in $89 \%$ as described above for 7 and as literature. ${ }^{1)}$

2-Azidoethyl 4-O-( $\beta$-D-galatopyranosyl)- $\beta$-D-glucopyranoside (9). To a solution of 7 (4.0 g, 5.68 mmol) in anhydrous methanol $(20 \mathrm{~mL})$ at $0{ }^{\circ} \mathrm{C}$ was added sodium methoxide $(320 \mathrm{mg}, 5.68 \mathrm{mmol})$. The reaction mixture was stirred for $3 \mathrm{hrs}$ at room temperature. Dowex cation exchange resin ( $\mathrm{H}$ form) was added to adjust the $\mathrm{pH}$ to 6-7 and filtered. The filtrate was evaporated to give a residue, which was purified by column chromatography $\left(\mathrm{SiO}_{2}\right)$ using chloroform-methanol (4:1) as eluent to afford 9 (2.240 g, 96\%). ${ }^{1} \mathrm{H}$ NMR $\left(\mathrm{CD}_{3} \mathrm{OD}\right) \delta: 4.35(\mathrm{~d}, 1 \mathrm{H}, J=7.5 \mathrm{~Hz}), 4.34(\mathrm{~d}, 1 \mathrm{H}, J=7.8 \mathrm{~Hz}), 4.02(\mathrm{t}, 1 \mathrm{H}$, $J=5.1 \mathrm{~Hz}), 3.98(\mathrm{dd}, 1 \mathrm{H}, J=5.7,5.1 \mathrm{~Hz}), 3.90(\mathrm{dd}, 1 \mathrm{H}, J=2.4,9.3 \mathrm{~Hz}), 3.87(\mathrm{dd}, 1 \mathrm{H}, J=8.1,5.1$ $\mathrm{Hz}$ ), 3.81 (br, $1 \mathrm{H}), 3.78-3.60(\mathrm{~m}, 2 \mathrm{H}), 3.62-3.44$ (m. $4 \mathrm{H})$. HR-MS (FAB) calcd. for $\mathrm{C}_{14} \mathrm{H}_{26} \mathrm{O}_{11} \mathrm{~N}_{3}$ 412.1577 found $412.1567[\mathrm{M}+\mathrm{H}]^{+}$.

2-Azidoethyl- $\beta$-D-glucopyranoside (8) was synthesized from 6 in $97 \%$ as described above for 9. ${ }^{1} \mathrm{H}$ NMR $\left(\mathrm{CD}_{3} \mathrm{OD}\right) \delta: 4.32(\mathrm{~d}, 1 \mathrm{H}, J=8.0 \mathrm{~Hz}), 3.86(\mathrm{dt}, 1 \mathrm{H}, J=10.8,4.4 \mathrm{~Hz}), 3.72(\mathrm{dd}, 1 \mathrm{H}, J=1.6$, $12.0 \mathrm{~Hz}), 3.66(\mathrm{dd}, 1 \mathrm{H}, J=4.4,10.0 \mathrm{~Hz}), 3.53(\mathrm{dd}, 1 \mathrm{H}, J=6.0,12.4 \mathrm{~Hz}), 3.45$ (dd, $1 \mathrm{H}, J=6.4,11.6$ $\mathrm{Hz}), 3.37$ (t, $1 \mathrm{H}, J=8.8 \mathrm{~Hz}), 3.33-3.25(\mathrm{~m}, 2 \mathrm{H}), 3.20(\mathrm{t}, 1 \mathrm{H}, J=9.2 \mathrm{~Hz}), 3.10(\mathrm{t} .1 \mathrm{H}, J=8.1 \mathrm{~Hz})$. HR-MS (FAB) calcd. for $\mathrm{C}_{8} \mathrm{H}_{15} \mathrm{O}_{6} \mathrm{~N}_{3} 249.0961$ found $250.0910[\mathrm{M}+\mathrm{H}]^{+}$.

2-aminoethyl 4- $\boldsymbol{O}$-( $\boldsymbol{\beta}$-D-galatopyranosyl)- $\boldsymbol{\beta}$-D-glucopyranoside (2). In the presence of $\mathrm{Pt}_{2} \mathrm{O}(240$ $\mathrm{mg}), 9(2.0 \mathrm{~g}, 4.86 \mathrm{mmol})$ in anhydrous methanol $(20 \mathrm{~mL})$ was charged with hydrogen balloon for $3 \mathrm{hrs}$ 
at room temperature. The reaction mixture was filtered, and the filtrate was evaporated to give a residue, which was purified on Sephadex-LH 20 with $\mathrm{MeOH}$ to afford 2 (188 mg, 84\%). ${ }^{1} \mathrm{H}$ NMR $\left(\mathrm{CD}_{3} \mathrm{OD}\right) \delta: 4.38(\mathrm{~d}, 1 \mathrm{H}, J=7.5 \mathrm{~Hz}), 4.36(\mathrm{~d}, 1 \mathrm{H}, J=7.8 \mathrm{~Hz}), 3.82(\mathrm{dd}, 1 \mathrm{H}, J=5.7,5.1 \mathrm{~Hz}), 3.80$ (dd, $1 \mathrm{H}, J=2.4,9.3 \mathrm{~Hz}), 3.72-3.46$ (m, $1 \mathrm{H}), 3.81$ (br, $1 \mathrm{H}), 3.42$ (dd, $1 \mathrm{H}, J=7.4,7.2 \mathrm{~Hz}), 2.78$ (t, 1 $\mathrm{H}, J=6.6 \mathrm{~Hz})$. HR-MS (FAB) calcd. for $\mathrm{C}_{14} \mathrm{H}_{27} \mathrm{O}_{11} \mathrm{~N} 385.1584$ found $386.1523[\mathrm{M}+\mathrm{H}]^{+}$.

2-aminoethoxyl- $\beta$-D-glucopyranoside (1) was synthesized from $\mathbf{8}$ in $86 \%$ as described above for $2 .{ }^{1} \mathrm{H}$ $\operatorname{NMR}\left(\mathrm{CD}_{3} \mathrm{OD}\right) \delta: 4.34(\mathrm{~d}, 1 \mathrm{H}, J=10.8 \mathrm{~Hz}), 3.95(\mathrm{~m}, 1 \mathrm{H}), 3.82-3.70(\mathrm{~m}, 2 \mathrm{H}), 4.55(\mathrm{dd}, 1 \mathrm{H}, J=6.0$, 16.4 Hz), 3.37-3.06 (m, $6 \mathrm{H})$. HR-MS (FAB) calcd. for $\mathrm{C}_{8} \mathrm{H}_{17} \mathrm{O}_{6} \mathrm{~N} 223.1056$ found $224.1044[\mathrm{M}+\mathrm{H}]^{+}$.

\section{3. ${ }^{1} \mathrm{H}$ NMR characterization of $\mathrm{B9}$ and Glucose, lactose and sialyl lewis $\mathrm{x}$-modified B9}

The obtained glyco-polypeptides were confirmed by ${ }^{1} \mathrm{H}-\mathrm{NMR}$ spectra, in which sugar installation ratios were calculated by comparing the integration of protons of sugar linker and polypeptide backbone protons.

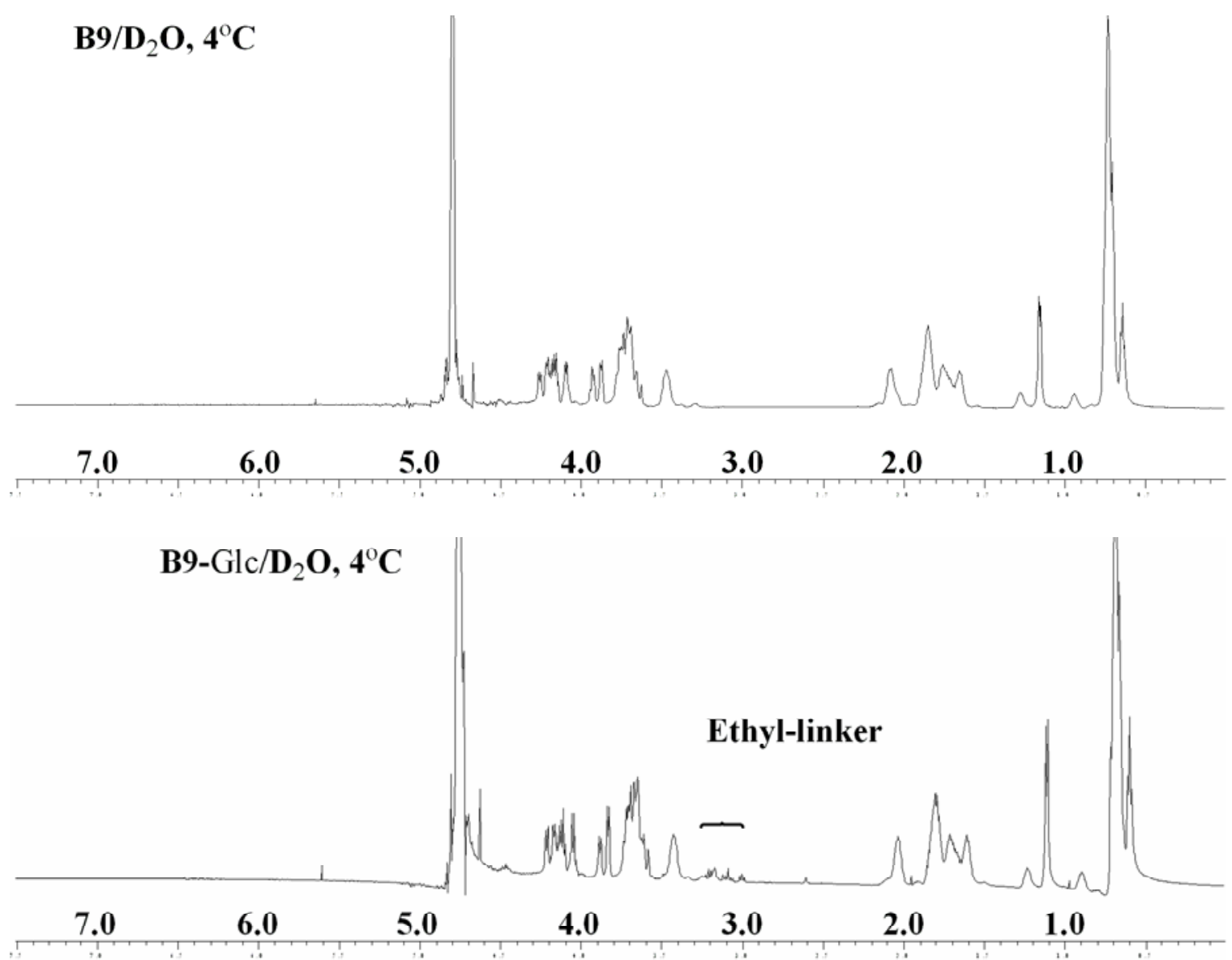



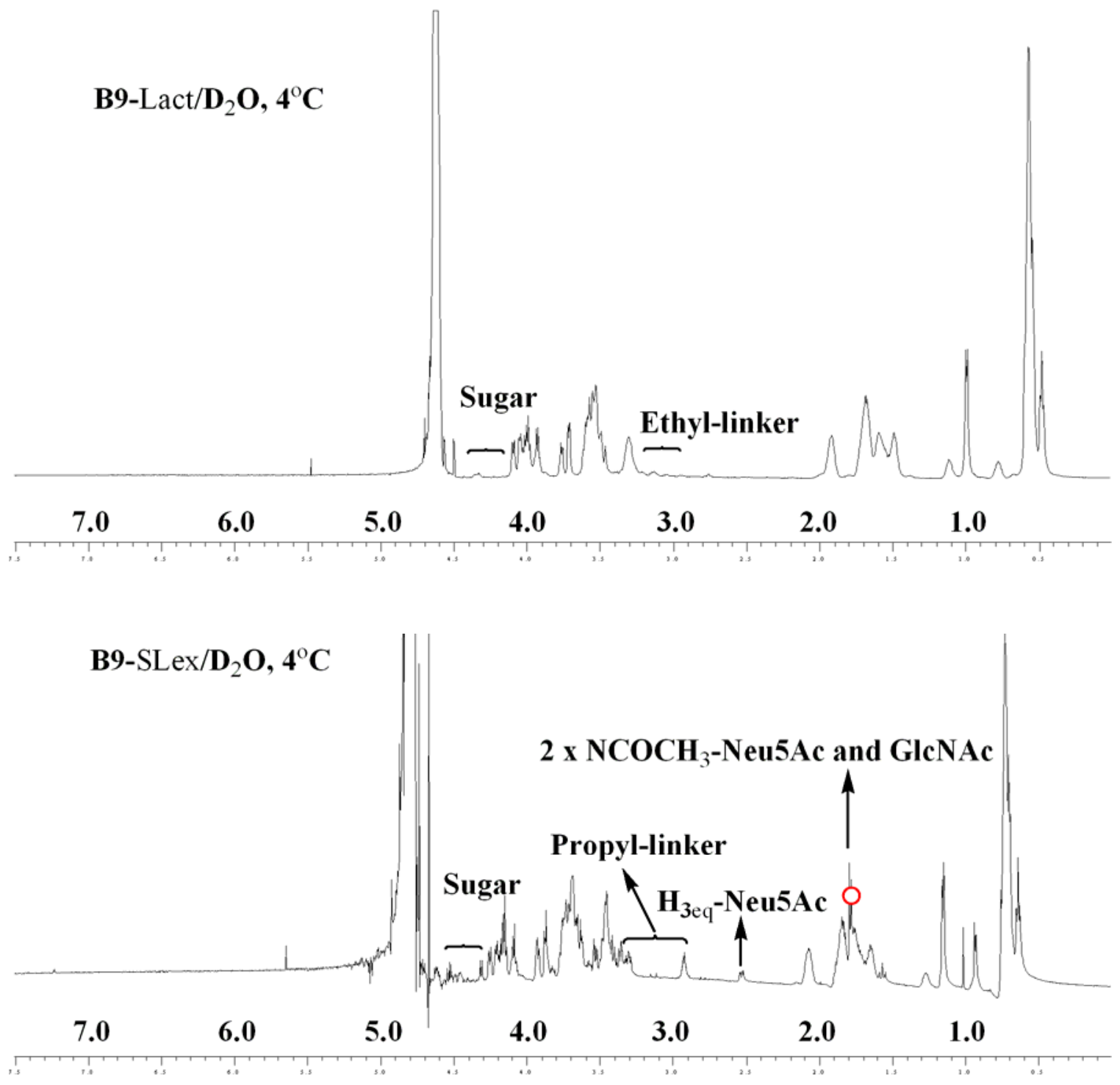

\section{References:}

1. A. Chernyak, G. Sharma, L. Kononov, P. Krishna, A. Levinsky, N. KochetkovAlla and V. R. Rao, Carbohydrate Research 223, 303-309, 1992. 